\title{
Macroporous Ultramicroelectrodes for Improved Electroanalytical Measurements
}

\author{
Rafael Szamocki,t,‡ Alexandra Velichko,§ Christian Holzapfel,§ Frank Mucklich,§ Serge Ravaine," \\ Patrick Garrigue, ${ }^{\dagger}$ Neso Sojic, ${ }^{\dagger}$ Rolf Hempelmann, ${ }^{\ddagger}$ and Alexander Kuhn ${ }^{\star, \dagger}$
}

Laboratoire d'Analyse Chimique par Reconnaissance Moléculaire, ENSCPB, 16 avenue Pey Berland, 33607 Pessac, France, Physikalische Chemie and Material Science Department, Universität des Saarlandes, 66123 Saarbrücken, Germany, and Centre de Recherche Paul Pascal, avenue Albert Schweitzer, 33600 Pessac, France

Recent work on the preparation of highly organized macroporous electrodes and nanoporous ultramicroelectrodes has been combined and extended to elaborate macroporous ultramicroelectrodes (UMEs) by template synthesis using colloidal crystals and following two different and complementary methods. On the one hand, arched porous UMEs were prepared, and on the other hand, cylindrical porous UMEs were obtained by using cavity UMEs. These macroporous UMEs have an active surface area which is up to 2 orders of magnitude higher compared to that of a classical disk UME as characterized by cyclic voltammetry. To study their analytical performance, the macroporous UMEs have been modified with a redox-active thiol and also a model bioelectrocatalytical system containing a redox mediator, a cofactor, and glucose-dehydrogenase. In both cases the electrochemical signal is amplified by up to 2 orders of magnitude, which increases significantly the analytical performance of such electrodes and therefore opens up new applications for this kind of miniaturized electrochemical system.

The miniaturization of electrochemical systems has been of high interest for applications for many years. For example, the preparation of miniaturized biofuel cells has already successfully been reported, ${ }^{1-3}$ and nanoelectrodes are now prepared and used as sensing units. ${ }^{4}$ However, often miniaturization implies that the signal-to-noise ratio for sensors or the power output for biofuel cells is decreased and is not sufficient for many applications. One possible solution for this problem is the artificial increase of the active surface area by using electrodes with porous surfaces. To use such electrodes as sensors, the resulting high surface area could be modified with molecular recognition units and should show significantly increased signals for monitoring the concentra-

* To whom correspondence should be addressed. Phone: +335400065 73. Fax: +33 5400027 17. E-mail: kuhn@enscpb.fr.

$\dagger$ ENSCPB.

‡ Physikalische Chemie, Universität des Saarlandes.

$\S$ Material Science Department, Universität des Saarlandes.

"Centre de Recherche Paul Pascal.

(1) Chen, T.; Barton, S. C.; Binyamin, G.; Gao, Z. Q.; Zhang, Y. C.; Kim, H. H.; Heller, A. J. Am. Chem. Soc. 2001, 123, 8630-8631.

(2) Heller, A. Phys. Chem. Chem. Phys. 2004, 6, 209-216.

(3) Willner, I.; Katz, E. Angew. Chem., Int. Ed. 2000, 39, 1180-1218.

(4) Ballesteros Katemann, B. Schuhmann, W. Electroanalysis 2002, 14, 2228.

10.1021/ac0615854 CCC: $\$ 37.00$ (C) 2007 American Chemical Society Published on Web 12/13/2006 tion of analyte molecules. We have recently proposed a bioelectrocatalytic chain that might be an interesting candidate for the modification of such a high surface area microelectrode, thus allowing the analysis of biomolecules such as glucose, glutamate, or lactate with a better precision and sensitivity. ${ }^{5-7}$ The signal increase already observed for porous macroelectrodes is promising, ${ }^{8}$ and therefore, a similar approach should also be used for the design of porous ultramicroelectrodes (UMEs) as miniaturized electrochemical sensors with a good signal-to-noise ratio.

In recent work the preparation of macroporous electrodes by using colloidal crystal templating has already been reported, ${ }^{8-13}$ and comparable methods have also been used for the synthesis of photonic crystals by several authors. ${ }^{14-18}$ The results of the procedure were electrodes with interconnected face-centered cubic ordered monodisperse pores (Figure 1A). The 3D analysis with FIB nanotomography of the complex electrode surface (Figure 1B) has proven that all pores are interconnected. ${ }^{19}$ The active surface area was characterized by cyclic voltammetry, and an increase of up to 2 orders of magnitude compared to that of a flat electrode has been obtained. These macroporous electrodes could be modified by a redox mediator and an enzymatic cofactor $\left(\mathrm{NAD}^{+}\right)$, thus allowing the oxidation of glucose in the presence

(5) Mano, N.; Peyrou, P.; Kuhn, A. Electroanalysis 2001, 12, 770-774.

(6) Mano, N.; Kuhn, A.; Menu, S.; Dufourc, E. J. Phys. Chem. Chem. Phys. 2003, 5, 2082-2088.

(7) Mano, N.; Thienpont, A.; Kuhn, A. Electrochem. Commun. 2001, 3, 585589

(8) Szamocki, R.; Reculusa, S.; Ravaine, S.; Bartlett, P. N.; Kuhn, A.; Hempelmann, R. Angew. Chem., Int. Ed. 2006, 45, 1317-1321.

(9) Bartlett, P. N.; Baumberg, J. J.; Birkin, P. R.; Ghanem, M. A.; Netti, M. C. Chem. Mater. 2002, 14, 2199-2208.

(10) Ben-Ali, S.; Cook, D. A.; Evans, S. A. G.; Thienpont, A.; Bartlett, P. N.; Kuhn, A. Electrochem. Commun. 2003, 5, 747-751.

(11) Ben-Ali, S.; Cook, D. A.; Bartlett, P. N.; Kuhn, A. J. Electroanal. Chem. 2005 , 579, 181-187.

(12) Bartlett, P. N.; Birkin, P. R.; Ghanem, M. A. Chem. Commun. 2000, 17, 1671-1672.

(13) Wang, C.; Yang, C.; Song, Y.; Gao, W.; Xia, X. Adv. Funct. Mater. 2005 , 15, 1267-1275.

(14) Velev, O. D.; Tessier, P. M.; Lenhoff, A. M.; Kaler, W. W. Nature 1999, 401, 548.

(15) Xu, L.; Zhou, W. L.; Frommen, C.; Baughman, R. H.; Zakhidov, A. A.; Malkinski, L.; Wang, J. Q.; Wiley, J. B. Chem. Commun. 2000, 997-998.

(16) Braun, P. V.; Wiltzius, P. Adv. Mater. 2001, 13, 482-485.

(17) Braun, P. V.; Wiltzius, P. Nature 1999, 402, 603-604.

(18) Juárez, B. H.; Golmayo, D.; Postigo, P. A.; López, C. Adv. Mater. 2004, 16, $1732-1736$.

(19) Szamocki, R.; Velichko, A.; Holzapfel, C.; Lasagni, A.; Mücklich, F.; Ravaine, S.; Kuhn, A.; Hempelmann, R. Manuscript in preparation. 

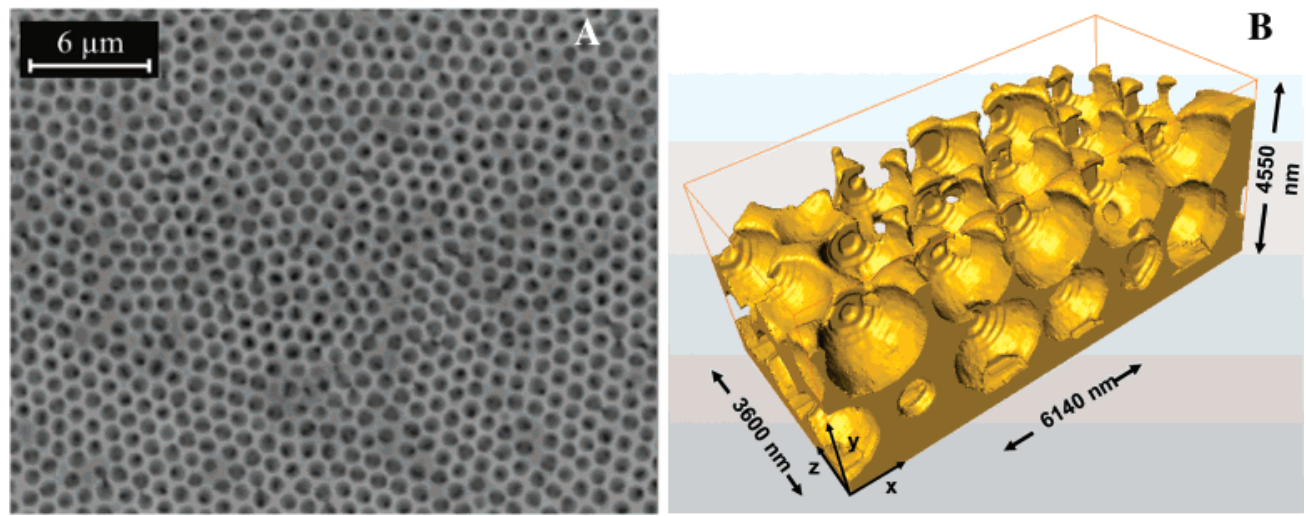

Figure 1. (A) SEM image of a macroporous gold electrode with a pore diameter of $1100 \mathrm{~nm}$ and $7 / 2$ pore layers obtained with a template of five layers of $1100 \mathrm{~nm}$ silica particles using the Langmuir-Blodgett technique. (B) Reconstruction of the real 3D gold structure of a macroporous electrode with a thickness of $3 / 2$ layers and a pore diameter of $1100 \mathrm{~nm}$. The reconstruction has been calculated from serial SEM images of FIB cross-sections performed every $50 \mathrm{~nm}$.

of glucose dehydrogenase (GDH) inside the porous system. Thereby, the signal for the glucose oxidation was increased by 1 or 2 orders of magnitude in the same way as the active surface area.

In parallel to this work the preparation of mesoporous ultramicroelectrodes by template synthesis and their use in analytical chemistry have been described by Denuault et al. ${ }^{20,21}$ Hexagonalphase liquid crystals made of amphiphilic molecules have been used as a template for the electrodeposition of noble metals, and the resulting electrode material has a surface area which is about 2 or 3 orders of magnitude higher than that of a classic ultramicroelectrode. ${ }^{22}$ Unfortunately, it is difficult with this technique to tune the pore diameter, which is very small when liquid crystals are used as a template. The maximum pore diameter that can be obtained is about $10 \mathrm{~nm}$ and is generally much too small to allow biomolecules and especially enzymes to enter the pores.

To circumvent this problem, we present in the following work a combination of the two concepts described above. Instead of mesoporous ultramicroelectrodes, we prepared macroporous ultramicroelectrodes by colloidal crystal templating and studied their use for bioelectrocatalytic reactions. Two strategies were followed to elaborate the macroporous ultramicroelectrodes (see Figure 2): the first is the deposition of a colloidal crystal on the flat polished glass surface containing the gold microdisk. After electrodeposition and dissolution of the template with hydrofluoric acid, the resulting porous gold had an arched shape because of the diffusion profile around the UME during the electrodeposition step (type A electrode). In a second approach we explored the preparation of cylindrical porous structures by using cavity ultramicroelectrodes (CMEs). ${ }^{23,24}$ These CMEs have been produced by controlled etching of the embedded gold wire of a UME to get a cylindrical cavity above the underlying electrode. This

(20) Imokawa, T.; Williams, K.J.; Denuault, G. Anal. Chem. 2006, 78, 265271.

(21) Evans, S. A. G.; Elliott, J. M.; Andrews, L. M.; Bartlett, P. N.; Doyle, P. J.; Denuault, G. Anal. Chem. 2002, 74, 1322-1326.

(22) Attard, G. S.; Bartlett, P. N.; Coleman, N. R. B.; Elliot, J. M.; Owen, J. R.; Wang, J. H. Science 1997, 278, 838-840.

(23) Vivier, V.; Cachet-Vivier, C.; Mezaille, S.; Wu, B. L.; Cha, C. S.; Nedelec, J.-Y.; Fedoroff, M.; Michel, D.; Yu, L. T. J. Electrochem. Soc. 2000, 147 4252-4262.

(24) Vivier, V.; Cachet-Vivier, C.; Wu, B. L.; Cha, C. S.; Nedelec, J.-Y.; Yu, L. T. Electrochem. Solid-State Lett. 1999, 2, 385-387.

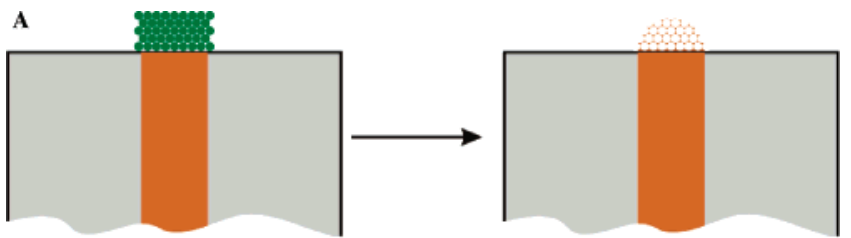

B

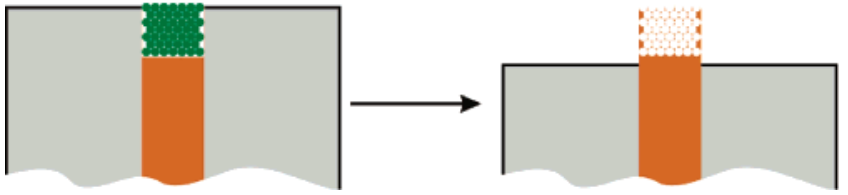

Figure 2. Schematic synthesis of the two types of macroporous UMEs: (A) arched type A electrode; (B) cylindrical type B electrode. Key: green, initial silica beads serving as a template; orange, gold metal; gray, glass sealing.

cavity was filled with a colloidal crystal, and gold was deposited through this template. By etching with HF not only the template but also the surrounding glass has been dissolved, and a cylindrical macroporous electrode is readily obtained (type B electrode).

In both cases the template has been formed by controlled evaporation of a diluted suspension of monodisperse spherical silica colloids. With this method the beads self-assemble during the evaporation process and form in the ideal case a face-centered cubic (fcc) colloidal crystal, a so-called artificial opal. Practically, this technique leads usually to structures showing some defects. During the subsequent electrodeposition, gold grows through the colloidal crystal, and after the dissolution of the colloidal particles, UMEs with ordered pores are obtained.

These procedures result in two different types of macroporous UMEs which have been characterized with respect to their electroactive surface area and their use as original systems for analytical measurements with increased efficiency.

\section{EXPERIMENTAL SECTION}

Reagents. All solutions were prepared using Milli-Q water. Cysteamine (Fluka), tetraethoxyorthosilicate (Sigma), hydrofluoric acid (48 wt \%, Aldrich), potassium iron(III) hexacyanoferrate (Fluka), calcium chloride (Sigma), Tris (Merck), NADH (Sigma), and $\mathrm{NAD}^{+}$(Sigma) were obtained in reagent grade and used as 
received without any further purification. Glucose dehydrogenase (GDH) from Bacillus megaterium (Jülich Fine Chemicals) was received with an activity of 76 units $/ \mathrm{mg}$. For the gold deposition a commercial gold-plating bath (ECF-60) from Technic Inc. was used. 4-(Ferrocenylcarbonyloxy) butanethiol was synthesized by esterification of ferrocenecarboxylic acid (Sigma) and 4-bromobutanol (Sigma), conversion of the bromide to a thioacetate with sodium thioacetate (Sigma), and, finally, mild hydrolysis of the thioacetate with sodium carbonate. ${ }^{25}$ For preparation of the Tris buffer corresponding aliquots were dissolved in water and the $\mathrm{pH}$ was adjusted by adding concentrated $\mathrm{HNO}_{3}$. (4-Carboxy-2,5,7trinitro-9-fluorenylidene) malononitrile (TNF) was synthesized as described in ref 26 and was used as a $5 \mathrm{mM}$ solution in Tris buffer. Monodisperse silica colloids (diameter $620 \mathrm{~nm}$ ) were prepared using the Stöber synthesis ${ }^{27}$ by hydrolysis of tetraethoxyorthosilicate.

Procedures. Cyclic voltammetry (CV), differential pulse voltammetry (DPV), and electrodeposition were carried out in a conventional cell with an Autolab PGSTAT 12 potentiostat with a low-current module at ambient temperature $\left(20 \pm 2{ }^{\circ} \mathrm{C}\right)$. All potentials were measured against a saturated $\mathrm{Ag} / \mathrm{AgCl}$ (BAS) reference electrode, and the counter electrode was a platinum wire. All scans were started at the negative end of the potential range. Parameters for DPV were chosen as follows: modulation time $t_{\mathrm{m}}=0.05 \mathrm{~s}$, interval time $t_{\mathrm{i}}=0.5 \mathrm{~s}$, modulation amplitude $E_{\mathrm{m}}$ $=25 \mathrm{mV}$, and step potential $E_{\mathrm{s}}=5 \mathrm{mV}$. SEM images were recorded by a JEOL JSM 5200 microscope.

For the preparation of the UMEs $20 \mu \mathrm{m}$ gold wires (Goodfellow) were sealed in a borosilicate glass tube using a heating spiral and connected to a silver wire by silver conductive paint. ${ }^{28-30}$ During the melting the glass tube was kept under vacuum using a water jet pump to ensure a perfect sealing of the gold wire. By polishing the final tip with emery paper $(25,3,1$, and $0.3 \mu \mathrm{m}$ grades, Thorlabs LFG03P), smooth microelectrode disks were obtained. After this the UMEs were cleaned with purified water in ultrasound.

Type A Electrodes. The disk ultramicroelectrodes were first modified with cysteamine to get a hydrophilic gold surface and were then fixed into the narrow end of a pipet tip using nail varnish. Afterwards the silica suspension ( $0.5 \mathrm{wt} \%)$ was poured into the pipet tip and slowly dried in a water vapor saturated atmosphere. After this evaporation step the gold electrolyte was poured into the tip, which acted at the same time as an electrochemical cell. After $10 \mathrm{~min}$, which allowed the gold solution to penetrate the whole colloidal crystal, the counter electrode and the reference electrode were fixed in this cell and a potential of $-0.66 \mathrm{~V}$ vs $\mathrm{Ag} / \mathrm{AgCl}$ was applied to the UME. During the deposition of gold through the template using chronoamperometry the thickness of the porous gold was controlled by following current oscillations and the global charge flow. ${ }^{8}$ After the electrodeposition the pipet tip was removed by dissolution of the

(25) Chidsey, C. E. D.; Bertozzi, C. R.; Putvinski, T. M.; Mujce, A. M. J. Am. Chem. Soc. 1990, 112, 4301-4306.

(26) Mano, N. Ph.D. Thesis, University Bordeaux, 2001

(27) Stöber, W.; Fink, A.; Bohn, E. J. J. Colloid Interface Sci. 1968, 26, 62-69.

(28) Howell, J. O.; Wightman, R. M. Anal. Chem. 1984, 56, 524-529.

(29) Denuault, G. Chem. Ind. 1996, 18, 678-680.

(30) Štulik, K.; Amatore, C.; Holub, K.; Mareček, V.; Kutner, W. Pure Appl. Chem. 2000, 72, 1483-1492.

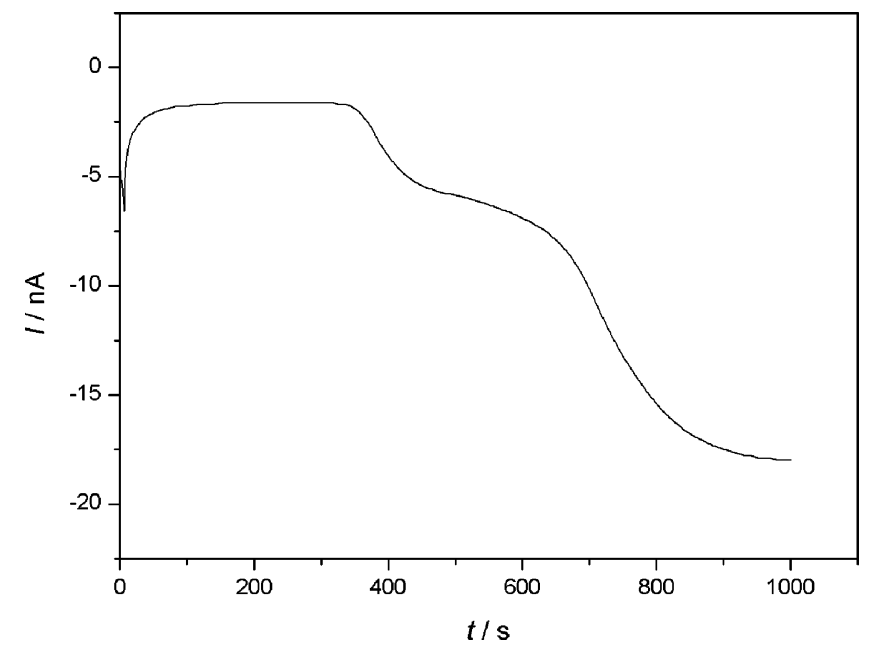

Figure 3. Current-time curve during the electrodeposition of a macroporous type B electrode. The electrodeposition was stopped in the third local current minimum, resulting in a porous layer with a thickness of $5 / 2$ pore layers.

varnish with acetone, and finally the porous UMEs were obtained by dissolving the colloids with $5 \mathrm{wt} \%$ hydrofluoric acid for $5 \mathrm{~min}$.

Type B Electrodes. First a cavity in the disk UMEs was formed by dissolving the gold wire with aqua regia for $15 \mathrm{~min}$, resulting in a cavity ultramicroelectrode with a depth of about 30 $\mu \mathrm{m} .{ }^{23,24}$ The rest of the procedure for obtaining this type of electrode was the same as for the type A structure, but after the pipet tip was filled with the colloids the cell was exposed to ultrasound to allow the suspension to penetrate the cavity and to remove air bubbles. After gold electrodeposition the electrodes were treated with $\mathrm{HF}$ for a longer time compared to the type A electrodes $(20 \mathrm{~min}$ ) to dissolve not only the colloidal crystal but also a part of the isolating glass capillary to uncover the cylindrical porous ultramicroelectrode.

Electrode Modification. Before modification the electrodes were cleaned by cycling the potential 20 times in $0.5 \mathrm{M} \mathrm{H}_{2} \mathrm{SO}_{4}$ between 0 and $+1.5 \mathrm{~V}$ vs $\mathrm{Ag} / \mathrm{AgCl}$ followed by 20 cycles in Tris buffer to remove the adsorbed sulfate ions. Then the mediator TNF was adsorbed on the electrode surface by dipping the electrodes in a $5 \mathrm{mM}$ solution of TNF in Tris buffer overnight. ${ }^{26}$ For the activation of the mediator the modified electrodes were cycled once between +0.3 and $-0.5 \mathrm{~V} \mathrm{vs} \mathrm{Ag} / \mathrm{AgCl}$ in Tris buffer. The modification with 4-(ferrocenylcarbonyloxy) butanethiol was achieved in the same way but using a $10 \mathrm{mM}$ ethanol solution of the molecule. ${ }^{25}$

\section{RESULTS AND DISCUSSION}

During the electrodeposition at constant potential temporary oscillations in the reduction current have been observed (Figure 3) and can be explained by an oscillating active electrode surface during the growth of the gold through the template. Such oscillations have already been described for the preparation of macroporous electrodes with a geometric area in the $\mathrm{cm}^{2}$ range using the Langmuir-Blodgett technique for building up the template. This allowed a precise control of the thickness of the macroporous material. ${ }^{8}$ However, the amplitude of this phenomenon is much less pronounced in the case of UMEs due to a higher defect density in colloidal crystals obtained by the evapora- 

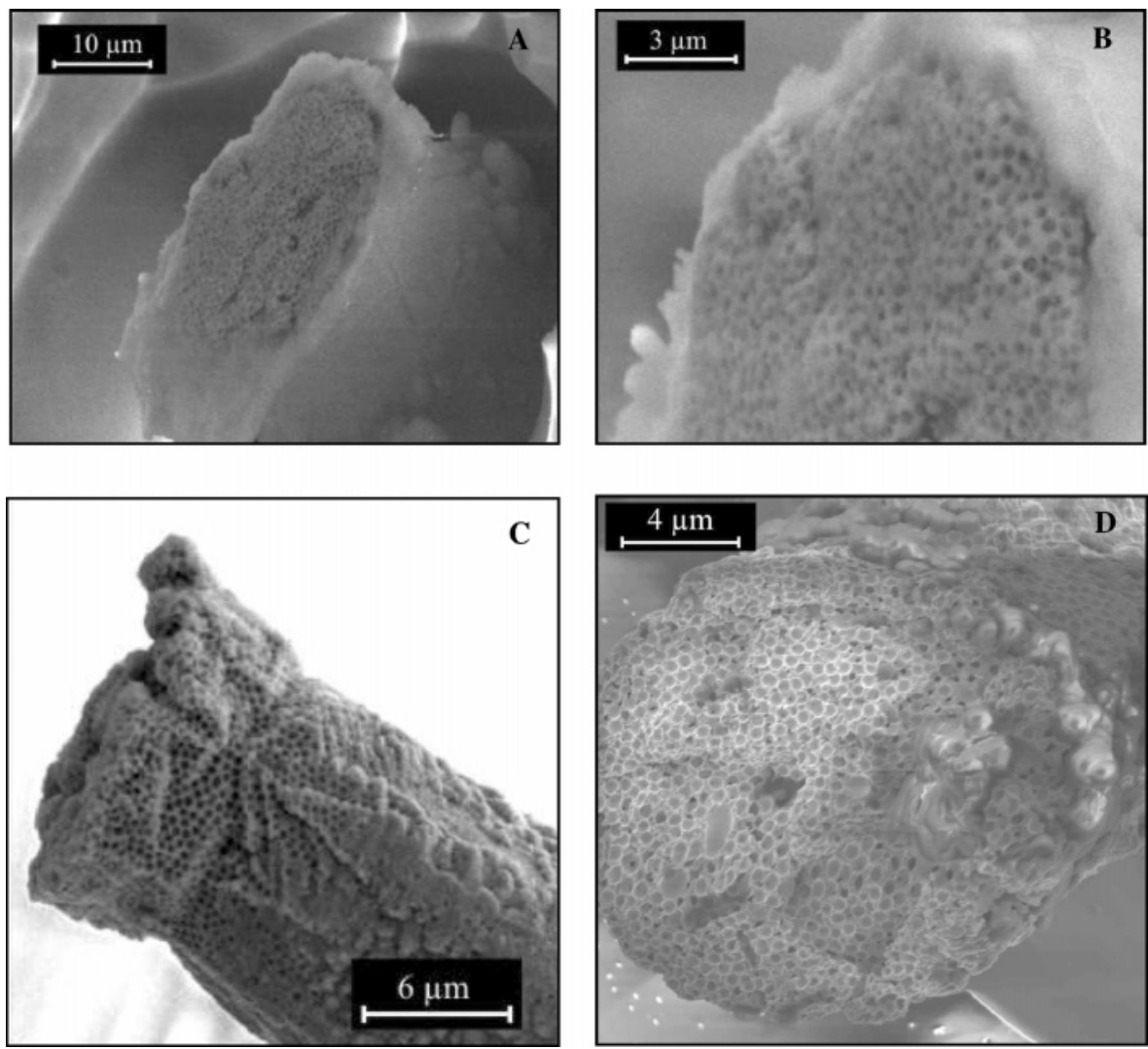

Figure 4. SEM images of macroporous ultramicroelectrodes: (A, B) arched type A UME at different magnifications; (C, D) cylindrical type B UME, side view (C) and top view (D).

tion method and also the bigger influence of border effects. Indeed for type A electrodes the growth of the metal layer is not strictly perpendicular to the electrode surface, at least for longer times, leading therefore to oscillations that are locally out of phase and finally cancel each other. In the case of the type B electrodes the oscillating overall reduction current increased with time. This could be explained by a change of the relative cavity depth during the process. In the beginning of the electrochemical deposition, the cavity is quite deep and the ion flow toward the electrode surface is hindered. When the cavity is filled with metal, the depth decreases and the diffusion is less and less disturbed, leading to a current increase. Also only three or four oscillations could be observed. As one full period of current oscillation corresponds to an advancement of the metal deposit by a thickness of exactly one bead layer (or two half-layers), ${ }^{8}$ four oscillations allow a thickness control of the porous material only until eight half-layers. The half-layer nomenclature has already been adopted in previous publications, $8,10,11$ because usually we stop the electrodeposition at half-height of a bead layer, thus generating in the last layer a completely open pore with a maximum diameter of the exposed section. The total thickness can be therefore expressed as the number of pore layers + (the last) half-pore-layer, or everything is converted in half-pore-layers (for example, 3 complete pore layers $+1 / 2$ pore layer $=7 / 2$ pore layers). For thicker porous electrodes the thickness has to be controlled by charge flow or the deposition time.

The morphology of the porous UMEs has been characterized by SEM. In Figure 4A,B an arched type A macroporous UME is shown. The gold microwire which is still embedded in a thin glass film for short treatment times with HF shows a complete and homogeneous coverage of the disk surface with closely packed monodisperse pores. The pore diameter correlates exactly with the diameter of the silica particles in the template. A closer look at the pores in the right-hand figure reveals very small dark points, representing the interconnections between the spherical pores and indicating that the complete inner surface should be accessible for electrochemical reactions.

In the same way the cylindrical type B electrodes show a homogeneous and ordered structure. As expected, only the end of the outstanding gold wire is porous while the underlying part has no micropores. For reasons of clarity of the illustration an unusually long porous cylinder has been depicted in Figure 4C, where the number of pore layers can no longer be controlled by the current oscillations mentioned above, but by measuring the charge flow during the electrodeposition. Compared to the arched electrodes, this type has no glass shell due to the longer HF dissolution step after electrodeposition. The pores can be seen both along the side surface and on the tip surface, thus indicating that the whole upper part of the cylinder is porous.

The increase of the surface activity of the macroporous UMEs has been characterized by using cyclic voltammetry in $0.5 \mathrm{M}$ sulfuric acid (Figure 5). The charge consumed during the surface oxidation and reduction was calculated by integration of the gold oxide stripping peak. The measured charge under the stripping peak was compared with that obtained for polished UMEs which have been etched in the same way as the porous ones. For both types of porous electrodes the measured charge and therefore the active surface area increases dramatically on going from a 

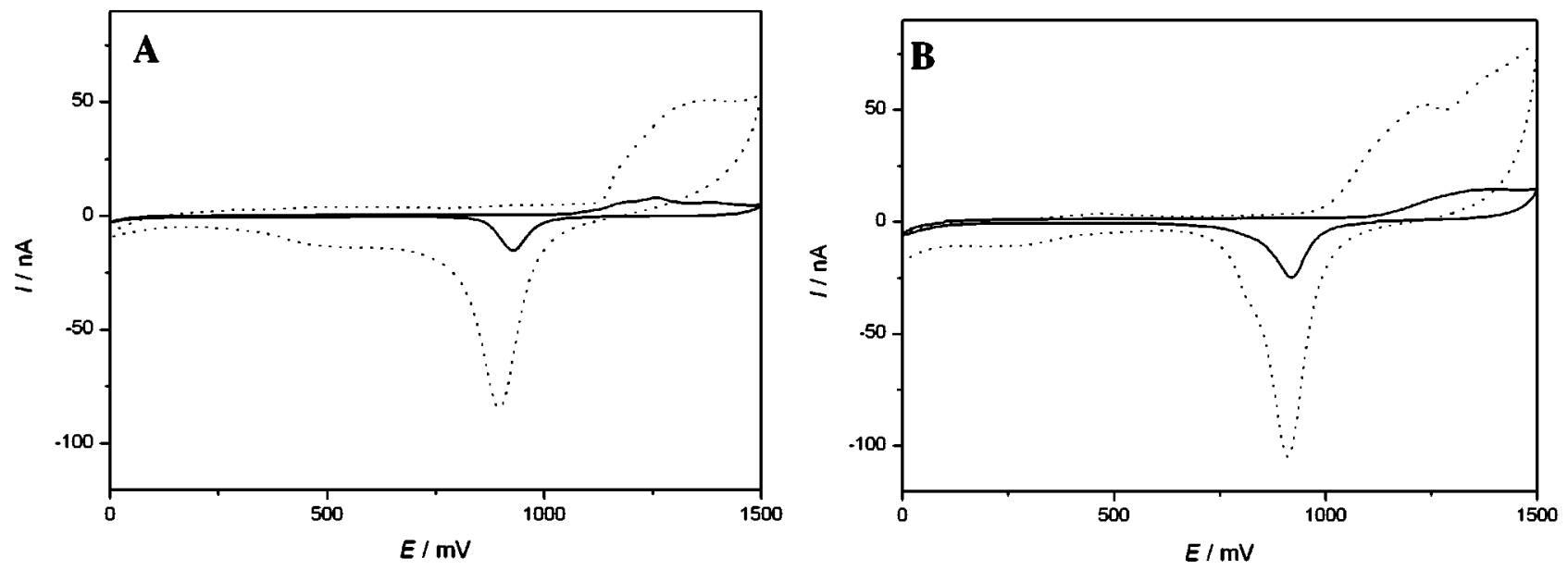

Figure 5. Cyclic voltammograms of macroporous UMEs (5/2 pore layers, dotted lines) compared to nonporous UMEs (solid lines) in $0.5 \mathrm{M}$ $\mathrm{H}_{2} \mathrm{SO}_{4}\left(v=100 \mathrm{mV} \cdot \mathrm{s}^{-1}\right)$ : (A) type A UME; (B) type B UME.

flat to a porous UME. Assuming a specific charge of $386 \mu \mathrm{C} / \mathrm{cm}^{2}$ for the gold oxide reduction, ${ }^{10}$ the type A UME has a total active surface of $3.3 \times 10^{-2} \mathrm{~mm}^{2}$ and the type B electrode a total active surface area of $4.3 \times 10^{-2} \mathrm{~mm}^{2}$, whereas for the corresponding nonporous electrode a typical surface area of $3.4 \times 10^{-3} \mathrm{~mm}^{2}$ is measured. The latter value is too high compared to what would be expected theoretically for the flat section of such a microelectrode, but this is most likely due to the etching that has also been applied to these "reference" electrodes for the sake of comparison. The theoretical value of the relative surface enhancement between a flat and a type A porous electrode can be calculated on the basis of fundamental geometric considerations, supposing a closepacked structure:

$$
f=n \pi(4 / 3)^{1 / 2}
$$

with $f$ being the enhancement factor and $n$ the number of pore layers.

For the 5/2 pore layer electrode of Figure $5 \mathrm{~A}$ a theoretical $f$ value of 9.1 is obtained, which is in good agreement with the measured surface ratio of 9.7. This seems to indicate that the total inner surface area of the electrode is active and can be easily increased, depending on the thickness of the porous layer, by more than 1 order of magnitude. With respect to the type A UMEs, the type B UMEs show a slightly higher surface because the side surface of this electrode type is not insulated, which results in an additional active surface area.

Due to this increased surface area the macroporous UMEs are expected to show a higher activity for electrochemical reactions in general. The first redox reaction that has been tested was a typical redox couple with a fast kinetics, $\left[\mathrm{Fe}(\mathrm{CN})_{6}\right]^{4-} /[\mathrm{Fe}-$ $\left.(\mathrm{CN})_{6}\right]^{3-}$. When this couple is used on flat electrodes, a linear increase of the signal as a function of the active surface area, usually proportional to the geometric area, can be observed. Unlike flat electrodes, porous ones do not show such a behavior for a given geometric area (Figure 6A). The first observation is that the signals in Figure 6A do not show the sigmoidal shape that is typical for UMEs, but small peaks can be observed. This is due to the relatively fast scan rate that has been used. ${ }^{31,32}$ The second and more important observation is that an increase of the active surface area by using a porous structure shows only a small increase of the electrochemical signal. This can be explained by the fast kinetics of the redox couple which reacts completely at the outermost half-pore-layer before the ions are able to diffuse into the porous system. In other terms there should be no difference between the first and subsequent "steady-state" scans because everything which is in the pores stays in the pores and does not react. The concentration profile of the reacting species coming from the bulk solution drops down to zero at the outermost half-pore-layer due to the fast kinetics. This means that the inner porous surface is not useful in the electrochemical sense and not the complete surface area, but only the last half-porelayer can be used for the turnover. Thus, macroporous electrodes are of very limited interest for redox reactions with fast electrontransfer kinetics. In this case only the controlled roughness of the outer pore layer gives a slight increase in electrochemical signal (independent of the total number of pore layers) because of the curved pore structure.

However, for reactions of immobilized species and also slow reactions such as electroenzymatic reactions an increase of the signal can easily be obtained as shown in the following experiments (Figure 6B-D).

First, a ferrocene compound (4-(ferrocenylcarbonyloxy)butanethiol) was immobilized by a covalent bond to the gold surface using a thiol group leading, to a monolayer (SAM). The ferrocenemodified electrodes were characterized by differential pulse voltammetry in $0.1 \mathrm{M} \mathrm{HClO}_{4}$ to eliminate the also increasing contribution of the capacitive current on going from flat to porous electrodes (Figure 6B). The voltammogramms show a peak at $+550 \mathrm{mV}$ corresponding to the oxidation of $\mathrm{Fe}^{\mathrm{II}}$ to $\mathrm{Fe}^{\mathrm{III}}$ in the immobilized ferrocene. The signal of a porous type B electrode is increased by more than 1 order of magnitude compared to that of a nonporous one. This indicates that the whole inner surface area can be modified with redox-active species that might also be used as electrocatalysts.

(31) Bard, A. J.; Faulkner, L. R. Electrochemical methods, 1st ed.; Wiley: New York, 1980.

(32) Girault, H. H. Analytical and Physical Electrochemistry, 1st ed.; EPFL Press: Lausanne, Switzerland, 2004.

(33) Newton, M. R.; Morey, K. A.; Zhang, Y.; Snow, R. J.; Diwekar, M.; Shi, J.; White, H. S. Nano Lett. 2004, 4, 875-880. 

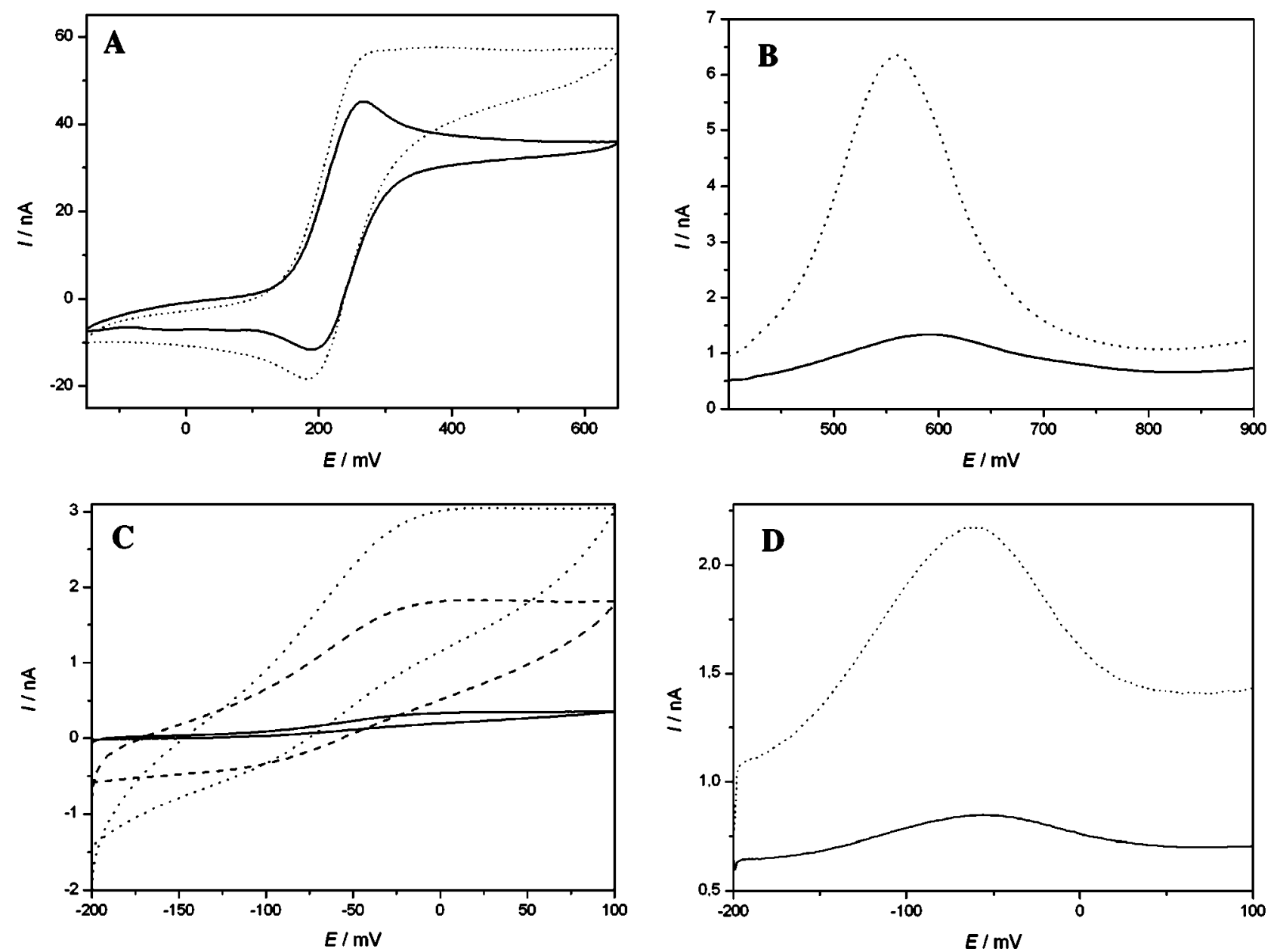

Figure 6. (A) Cyclic voltammograms of type $B$ UMEs in a solution of $5 \mathrm{mM} \mathrm{K} 4\left[\mathrm{Fe}(\mathrm{CN})_{6}\right]$ in $1 \mathrm{M} \mathrm{KNO}_{3}\left(v=100 \mathrm{mV} \cdot \mathrm{s}^{-1}\right)$ : solid line, flat UME; dotted line, porous UME (5/2 pore layers). (B) Differential pulse voltammograms of type B UMEs modified with a self-assembled monolayer of (4-(ferrocenylcarbonyloxy)butanethiol) in $0.1 \mathrm{M} \mathrm{HClO}_{4}\left(v=10 \mathrm{mV} \cdot \mathrm{s}^{-1}\right)$ : solid line, flat UME; dotted line, porous UME (5/2 pore layers). (C) Cyclic voltammograms of type A UMEs modified with TNF and NAD in a solution of $10 \mathrm{mM}$ glucose and $3 \mathrm{U} \cdot \mathrm{mL}^{-1} \mathrm{GDH}$ in $0.1 \mathrm{M}$ Tris buffer $\left(v=10 \mathrm{mV} \cdot \mathrm{s}^{-1}\right)$ : solid line, flat UME; dashed line, porous UME (3/2 pore layers); dotted line, porous UME (5/2 pore layers). (D) Differential pulse voltammograms of type B UMEs modified with TNF in a solution of $3.2 \mathrm{mM} \mathrm{NADH}$ and $0.2 \mathrm{M} \mathrm{CaCl}_{2}$ in $0.1 \mathrm{M} \mathrm{Tris} \mathrm{buffer}\left(v=10 \mathrm{mV} \cdot \mathrm{s}^{-1}\right)$ : solid line, flat UME; dotted line, porous UME (5/2 pore layers).

In previous work we already reported the catalytic activity of immobilized TNF for the electrooxidation of NADH. ${ }^{5-7}$ Since the $\mathrm{NADH} / \mathrm{NAD}^{+}$system is a cofactor for many enzymes, it can be used in combination with TNF and GDH to design a model system biosensor for the specific detection of glucose by amperometric measurements. This catalytic chain has already been used for the modification of $\mathrm{cm}^{2}$-sized macroporous electrodes, resulting in an increase of the glucose signal by roughly 2 orders of magnitude. ${ }^{8}$ In the following we show that also the entire active surface area of macroporous UMEs can be modified with this catalytic chain and that they can be successfully used as miniaturized biosensors with an improved signal. In a first step the mediator TNF was immobilized as a molecular monolayer by covalent binding through cyanide groups on the electrode surface. This monolayer was covered by a second monolayer of $\mathrm{NAD}^{+}$which was immobilized using an electrostatic $\mathrm{Ca}^{2+}$ bridge. ${ }^{5,6}$ This ion forms a complex with the carboxyl group of the mediator on one side and the phosphate groups of the $\mathrm{NAD}^{+}$on the other side. By using the so-modified UME as a working electrode in a solution containing GDH, the electrode is able to specifically detect glucose. Figure $6 \mathrm{C}$ shows the cyclic voltammogram of two macroporous type A electrodes and a flat disk UME modified with TNF and $\mathrm{NAD}^{+}$in a solution of $10 \mathrm{mM}$ glucose in $0.1 \mathrm{M}$ Tris buffer containing $3 \mathrm{U} \cdot \mathrm{mL}^{-1} \mathrm{GDH}$. Depending on the number of porous layers, the glucose signal can easily be increased by 1 or 2 orders of magnitude. This means that the absolute faradaic current signal for these UMEs is significantly improved; however, the contribution corresponding to the capacitive current increases in the same way, because it is also directly proportional to the active surface area. To circumvent this problem, differential pulse voltammetry can again be used like in the experiment of Figure $6 \mathrm{~B}$, eliminating the contribution of the capacitive current and leading finally to a net improvement of the performance of these porous electrodes in terms of sensitivity and detection limits. This is illustrated in Figure 6D for the electrocatalytic oxidation of $\mathrm{NADH}$ into $\mathrm{NAD}^{+}$. As can be seen, the use of DPV efficiently decreases the more or less constant signal of the capacitive current, allowing a full benefit from the increase in faradaic current on changing from a flat to a porous UME. 


\section{CONCLUSION}

We developed two complementary approaches for the elaboration of macroporous UMEs in a straightforward electrochemical template synthesis. These two types of electrodes could be useful for different applications, e.g., invasive measurements in biological cells with cylindrical UMEs or noninvasive in-touch measurements with arched macroporous UMEs. In comparison to liquid-crystaltemplated mesoporous UMEs, the signal increase is lower (2 orders of magnitude instead of 3 ), but the range of pore diameters is much more variable (50-1000 $\mathrm{nm}$ compared to $5-10 \mathrm{~nm}$ ), and therefore, macroporous UMEs might have an interesting and complementary range of applications. For example, they can easily be modified with enzymes, which is not possible for mesoporous UMEs because of the enzyme's diameter. These enzyme-modified UMEs with an increased active surface area, in combination with the elimination of the capacitive current by DPV, might open up important applications not only in electroanalytical chemistry, but also more generally in the field of miniaturized electrochemical devices such as biofuel cells. ${ }^{1-3}$ In addition a more detailed study of the coupled transport/reaction phenomena in these complex structures, analogous to what has been reported recently for similar electrodes ${ }^{33}$ needs to be performed to fully understand and optimize the behavior of these interesting tools.

\section{ACKNOWLEDGMENT}

We thank the DFG (SFB277), the DAAD, and the CNRS through the "Programme Matériaux" (A.K. and S.R.) for financial support. We are indebted to the French Ministry of Research for a Cotutelle grant and for financial support through the FrenchGerman exchange program "Procope".

Received for review August 24, 2006. Accepted October 26, 2006.

AC0615854 